\title{
Macrophage-derived human resistin exacerbates adipose tissue inflammation and insulin resistance in mice
}

\author{
Mohammed Qatanani, ${ }^{1}$ Nava R. Szwergold,1 David R. Greaves, ${ }^{2}$ \\ Rexford S. Ahima, ${ }^{1}$ and Mitchell A. Lazar ${ }^{1}$

\begin{abstract}
1Division of Endocrinology, Diabetes, and Metabolism, Department of Medicine, and Institute for Diabetes, Obesity, and Metabolism, University of Pennsylvania School of Medicine, Philadelphia, Pennsylvania, USA. ${ }^{2}$ Sir William Dunn School of Pathology, University of Oxford, Oxford, United Kingdom.
\end{abstract}

\begin{abstract}
Resistin is an adipokine that contributes to insulin resistance in mice. In humans, however, studies investigating the link between resistin and metabolic disease are conflicting. Further complicating the matter, human resistin is produced mainly by macrophages rather than adipocytes. To address this important issue, we generated mice that lack adipocyte-derived mouse resistin but produce human resistin in a pattern similar to that found in humans, i.e., in macrophages (humanized resistin mice). When placed on a high-fat diet, the humanized resistin mice rapidly developed accelerated white adipose tissue (WAT) inflammation, leading to increased lipolysis and increased serum free fatty acids. Over time, these mice accumulated lipids, including diacylglycerols, in muscle. We found that this resulted in increased $\operatorname{Pkc} \theta$ pathway activity, leading to increased serine phosphorylation of Irs-1 and insulin resistance. Thus, although the site of resistin production differs between species, human resistin exacerbates WAT inflammation and contributes to insulin resistance.
\end{abstract}

\section{Introduction}

The number of obese individuals worldwide has reached 2.1 billion, leading to an explosion of obesity-related health problems associated with increased morbidity and mortality $(1,2)$. The increase in the prevalence of obesity is strongly correlated with an increase in type II diabetes mellitus (T2DM), which has reached epidemic proportions in the United States $(3,4)$. A key etiological factor linking obesity to T2DM is insulin resistance $(5,6)$. The pathophysiological mechanisms connecting obesity to insulin resistance have received intense investigation in recent years and include ectopic lipid accumulation in liver and muscle, increased circulating serum FFAs, altered production of various adipocytederived factors (adipokines), and low-grade inflammation of white adipose tissue (WAT) resulting from chronic activation of the innate immune system (reviewed in ref. 7).

Of the described adipokines, resistin seems to assert its effects on both inflammatory and insulin signaling pathways (reviewed in ref. 8). Resistin, also known as found in inflammatory zone 3 (Fizz3) and adipocyte-specific secretory factor, belongs to a family of small, cysteine-rich secreted proteins (9-11). In rodents, evidence is accumulating that resistin impairs insulin sensitivity. Circulating levels of resistin are increased in obesity, and an increase in serum resistin levels has been shown to induce insulin resistance in several rat and mouse models (11-15). These studies suggest that hyperresistinemia causes insulin resistance and predisposes to T2DM. Consistent with the conclusions from gain-of-function studies, loss of resistin signaling improves insulin sensitiv-

Conflict of interest: The authors have declared that no conflict of interest exists. Nonstandard abbreviations used: DAG, diacylglycerol; GIR, glucose-infusion rate; GTT, glucose-tolerance test; HSL, hormone-sensitive lipase; LPL, lipoprotein lipase; MCP-1, monocyte chemotactic protein-1; PDE3b, phosphodiesterase 3b; PTH, parathyroid hormone; Retn, resistin; ser307, serine 307; SVF, stromal vascular fraction; T2DM, type II diabetes mellitus; WAT, white adipose tissue.

Citation for this article: J. Clin. Invest. 119:531-539 (2009). doi:10.1172/JCI37273. ity and glucose homeostasis in several rodent models $(11,16-18)$. The majority of in vivo studies show that resistin has a negative effect on insulin signaling in the liver, although more recent studies implicate a negative effect of resistin on insulin signaling in adipose as well as muscle tissues $(19,20)$.

Although animal studies have given us a great deal of understanding of the major roles that resistin plays in normal glucose homeostasis as well as the pathophysiology of insulin resistance in rodents, the role of resistin in humans is less certain. Some studies have shown significant relationships between resistin levels and obesity, insulin resistance, or T2DM (21-27). However, other studies failed to identify changes in resistin levels in one or more of these conditions (28-30). Intriguingly, recent studies in humans show a very strong and consistent association between resistin and inflammation and/or inflammatory diseases (31-34).

Given the tremendous potential value of translating the mouse data to the human, it is critical to determine whether levels of resistin are increased in human obesity, insulin resistance, and/or T2DM and whether hyperresistinemia is a contributing factor to these pathophysiological states in humans. However, translating the biology of murine resistin to the human situation is complicated by interspecies differences that exist between the major site of resistin production in rodents (exclusively from adipocyte tissue) and that in humans (mainly macrophages) $(11,30,35)$. To test the hypothesis that human resistin produced by monocytes and macrophages contributes to insulin resistance, we generated and characterized what we believe is a novel transgenic mouse model that has macrophagespecific expression of human resistin but lacks murine resistin ("humanized resistin mice"). In the setting of diet-induced obesity, insulin resistance and WAT inflammation were worsened in the humanized resistin mice. Thus, macrophage-derived human resistin is capable of exacerbating the pathophysiological consequences of obesity. 
A
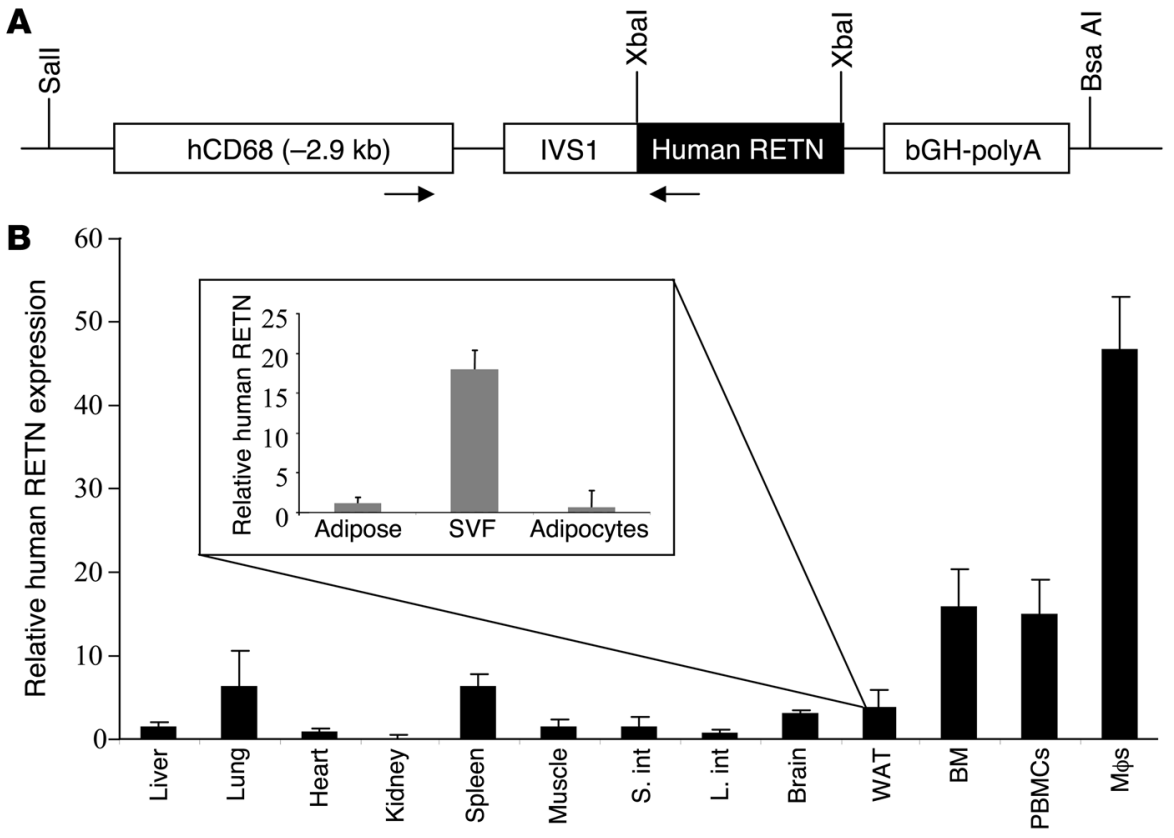

Figure 1

Derivation and initial characterization of humanized resistin mice. (A) CD68 promoter construct used to generate the mice. The CD68/human resistin cassette was excised using Sall and BsaAl, and the construct was injected into C57BL/6 fertilized eggs. Arrows represent the locations of the primers used to genotype the transgenic mice. (B) Expression profile of human resistin in different tissues in the humanized resistin mice as well as its expression profile in the different fractions of WAT (inset). Real-time RT-PCR was used to measure mRNA levels. Data are expressed as mean $\pm \mathrm{SEM} ; n=5$. S. int, small intestine; L. int, large intestine.

\section{Results}

Derivation and initial characterization of humanized resistin mice. Prior to generating the humanized transgenic mice, we showed that human resistin is able to activate the mouse resistin signaling pathway by inducing Socs3 gene expression in 3T3L1 adipocytes (Supplemental Figure 1; supplemental material available online with this article; doi:10.1172/JCI37273DS1) (19). To better understand the role of human resistin in the pathophysiology of insulin resistance and diabetes in humans, we derived transgenic mice on the C57BL/ 6 background with macrophage-specific expression of human resistin using the human CD68 promoter (Figure 1A) (36). Several independent lines were generated, 2 of which were established and bred to $\mathrm{C} 57 \mathrm{BL} / 6$ Retn $^{-1-}$ mice to generate mice that express human resistin but lack any expression of murine resistin (Retn) (20). The line we have studied the most, referred to hereafter as "humanized resistin mice," had circulating human resistin levels that are comparable to pathophysiological levels seen in humans $(48.75 \pm 7.50 \mathrm{ng} / \mathrm{ml})$ and were compared with their Retn-/- littermates ("controls," hereafter) $(37,38)$. The second line had higher human resistin levels $(141.72 \pm 11.45 \mathrm{ng} / \mathrm{ml})$ and were designated "the second line of humanized resistin mice." The humanized resistin mice were viable, were born in the expected Mendelian ratios (Supplemental Table 1), and appeared normal. As in humans, the highest level of tissue expression of human resistin was seen in macrophages, followed by high expression in bone marrow as well as peripheral blood mononuclear cells (Figure 2B). There was also significant expression in tissues that harbor large numbers of macrophages, such as spleen, lung, and fat. The second line of humanized resistin mice had similar tissue expression patterns (Supplemental Figure 2A). The expression of

\section{Figure 2}

Glucose homeostasis in humanized resistin mice. (A) Humanized resistin mice have decreased glucose tolerance and $(\mathbf{B})$ decreased insulin sensitivity. ${ }^{*} P<0.05$. Data are expressed as mean \pm SEM; $n=16$. human resistin in fat was predominantly in the stromal vascular fraction (SVF) compared with adipocytes, analogous to the situation in humans (Figure 1B) (39).

Lipid dysregulation in humanized resistin mice. The humanized resistin mice were studied under conditions of normal-chow feeding as well as on high-fat-diet feeding. There was no difference in weight

A
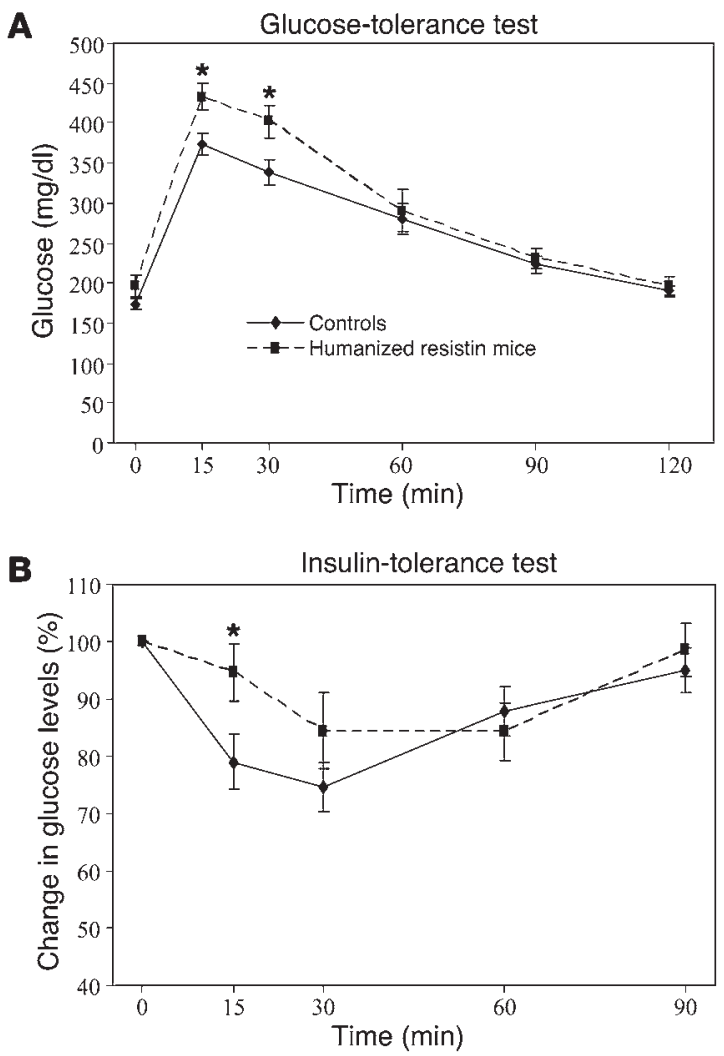
Table 1

Plasma metabolic profile of humanized resistin mice

$\begin{array}{lcc}\text { Genotype } & \text { Control mice } & \text { Humanized resistin mice } \\ \text { Weight }(\mathrm{g}) & 34.3 \pm 2.4 & 32.7 \pm 2.3 \\ \text { Glucose }(\mathrm{mg} / \mathrm{dl}) & 180.5 \pm 6.8 & 196.6 \pm 12.5 \\ \text { Insulin }(\mathrm{ng} / \mathrm{ml}) & 2.47 \pm 0.31 & 3.75 \pm 0.49^{\mathrm{A}} \\ \text { TGs }(\mathrm{mg} / \mathrm{dl}) & 68.0 \pm 3.8 & 55.5 \pm 2.1^{\mathrm{A}} \\ \text { XOL }(\mathrm{mg} / \mathrm{dl}) & 123.4 \pm 3.7 & 120.6 \pm 4.3 \\ \text { NEFA }(\mathrm{mEg} / \mathrm{l}) & 0.51 \pm 0.03 & 0.63 \pm 0.04^{\mathrm{A}} \\ \text { Glycerol }(\mathrm{mg} / \mathrm{dl}) & 0.025 \pm 0.001 & 0.029 \pm 0.002^{\mathrm{A}}\end{array}$

Humanized resistin mice fed a high-fat diet show serum profile indicative of insulin resistance and increased lipolysis. XOL, cholesterol; NEFA, nonesterified fatty acids. Data are expressed as mean $\pm \mathrm{SEM}$; $n=22$. ${ }^{A} P<0.05$.

gain or adiposity between the humanized resistin mice and the controls during the time frame of our experiments (Supplemental Figure 3). In the normal-chow-fed mice, there were also no appreciable differences in serum metabolic parameters, including serum triglycerides, FFAs, glycerol, and blood glucose and insulin levels (data not shown). However, with high-fat feeding, serum insulin levels were significantly higher in the humanized resistin mice, while serum glucose levels were higher, but the difference did not reach statistical significance (Table 1 ). Moreover, the humanized resistin mice had significantly higher serum levels of FFAs and glycerol, suggestive of increased lipolysis in these animals. Similar results were obtained with the second line of humanized resistin mice (Supplemental Figure 2B). The humanized resistin mice also had significantly lower triglycerides compared with the control animals, which may be explained by increased skeletal muscle lipoprotein lipase (Lpl) activity (see below).

Insulin resistance and decreased glucose disposal rate in the humanized resistin mice. There was no difference in glucose homeostasis between genotypes in the normal-chow-fed mice (Supplemental Figure 4). However, with high-fat feeding, glucose-tolerance test (GTT) revealed that the humanized resistin mice had impaired glucose metabolism compared with mice not expressing human resistin (Figure 2A). These mice also showed a general decrease in insulin sensitivity in the insulin tolerance tests (Figure 2B). The animals also showed higher levels of serum insulin during GTT, which reflects the higher glucose levels in these animals under GTT conditions and is consistent with their prediabetic state (Supplemental Figure 5).

In order to ascertain the specific tissue defect responsible for the insulin resistance seen in the humanized resistin mice, we performed hyperinsulinemic-euglycemic clamp studies (Figure 3). The glucose-infusion rate (GIR) required to maintain euglycemia in the humanized resistin mice was 39\% lower than in control littermates not expressing human resistin, indicating marked whole-body insulin resistance in this group. The exacerbated insulin resistance was due to decreased rate of disposal in the humanized resistin mice compared with controls (Figure 3A). Similar results were obtained with the second line of humanized resistin mice (Supplemental Figure 2, C and D). Given the defect in the rate of glucose disposal, we investigated the rate of glucose uptake in muscle and WAT, the main tissues responsible for glucose uptake, as well as insulin signaling in these tissues. Indeed, insulin-stimulated glucose uptake in skeletal muscle and WAT were significantly decreased (56\% and
$22 \%$, respectively; Figure 3B). This was accompanied by decreased tyrosine phosphorylation of Irs-1 (Figure 3C). Insulin-stimulated Akt phosphorylation was also attenuated in the muscle of the humanized resistin mice (Supplemental Figure 6). The resistance to insulin action in WAT was also evident by the decreased suppression of FFA release in response to insulin in the hyperinsulinemic clamp setting, as humanized resistin mice had serum FFA levels significantly higher than those of control mice not expressing human resistin $(0.304 \pm 0.033$ versus $0.208 \pm 0.009 \mathrm{mEq} / \mathrm{l})$.

Activation of the Pkc $\theta$ pathway in skeletal muscle in the humanized resistin mice. To understand the mechanism of the exacerbated skeletal muscle insulin resistance, we next evaluated gene-expression changes in the insulin-resistant muscles of the humanized resistin mice. No differences were observed in the expression of several inflammatory cytokines (Figure 4A). In contrast, Lpl expression was approximately 2 -fold higher in the skeletal muscles of the humanized resistin mice (Figure 4A), which was accompanied by a significant increase in Lpl enzyme activity (Figure 4B). Lpl is the rate-limiting enzyme for the uptake of triglyceride-derived fatty acids from the serum (40), which may explain why serum triglycerides were reduced in the humanized resistin mice (Table 1 and Supplemental Figure 2B). Increased skeletal muscle Lpl causes insulin resistance due to increased accumulation of intramyocellular lipids and lipid metabolites, including diacylglycerols (DAG) (41). Indeed, triglyceride levels were approximately 52\% higher in

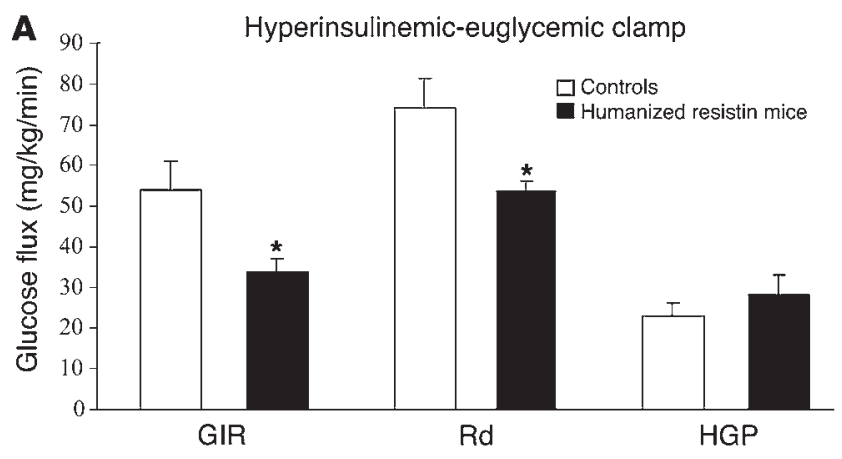

B

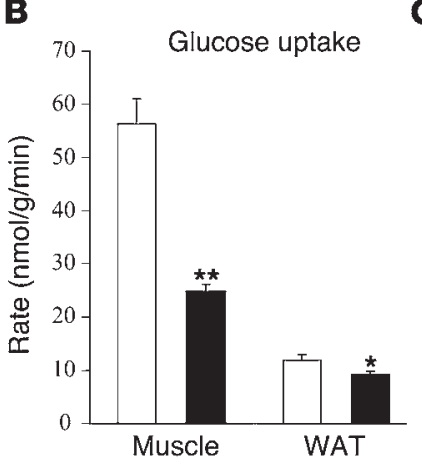

C Tyrosine phosphorylation

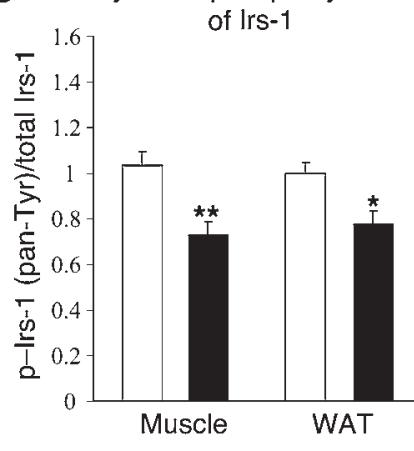

\section{Figure 3}

Humanized resistin mice are insulin resistant. (A) Hyperinsulinemiceuglycemic clamp analysis of humanized resistin mice versus Retn ${ }^{-/}$ controls. (B) Rate of insulin-stimulated glucose uptake in muscle and WAT. (C) Insulin-stimulated tyrosine phosphorylation of IRS-1 in muscle and WAT normalized to controls. Rd, rate of glucose disposal; HGP, hepatic glucose production; pan-Tyr, pan tyrosine. ${ }^{*} P<0.05$; ${ }^{* *} P<0.01$. Data are expressed as mean \pm SEM; $n=8$. 

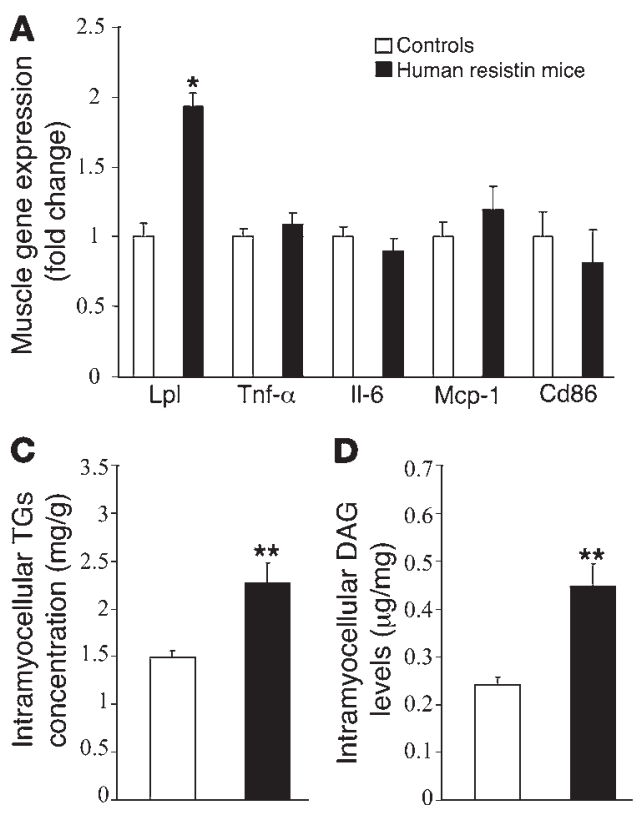

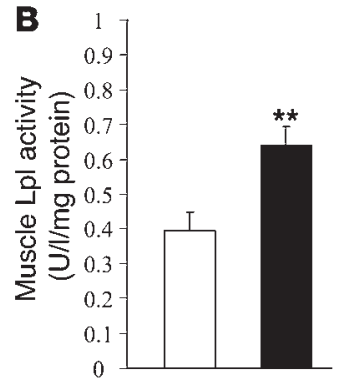

E

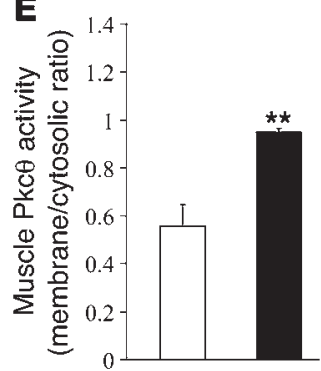

Figure 4

Increased lipid accumulation in the humanized resistin mice. (A) Gene-expression analysis in muscle of humanized resistin mice compared with control mice. (B) Heparinreleased muscle Lpl activity per mg protein. (C) Intramyocellular triglycerides concentration in skeletal muscle. (D) Intracellular muscle DAG levels. (E) Muscle Pkc0 activity expressed as membrane/cytosol ratio of muscle Pkc $\theta$ protein levels. TGs, triglycerides. ${ }^{*} P<0.05 ;{ }^{*} P<0.01$. Data are expressed as mean \pm SEM; $n=9$.

in the expression of phosphodiesterase 3b (Pde3b), the major enzyme responsible for the breakdown of cAMP and hence attenuation of hormone-sensitive lipase (Hsl) activity in fat cells in these animals. This might be secondary to the increase in Tnf- $\alpha$ in the WAT of the humanized resistin mice and is likely to have contributed to their increased lipolysis (Table 1) (46). The increase in Cd68 expression in the WAT of the humanized resistin mice indicated an increase in the accumulation of macrophages in this tissue. Indeed, immunohistochemical analysis demonstrated that humanized resistin mice had higher accumulation of WAT macrophages (Figure 5B). This is likely related to the increased expression of Mcp-1, which contributes to macrophage infiltration into $\operatorname{WAT}(47,48)$ and has been shown to be induced in endothelial cells treated with human resistin (49). One of the mechanisms by which inflammation leads to impairment in insulin signaling is through the inhibitory phosphorylation of Irs-1 on serine 307 (ser307) $(50,51)$. Consistent with the increased the muscle of humanized resistin mice (Figure 4C). Consistent with this, skeletal muscle DAG in humanized resistin mice on high-fat diets was approximately $85 \%$ higher than in controls (Figure 4D). DAG activates $\mathrm{Pkc} \theta$, which impairs insulin signaling by increasing the inhibitory serine phosphorylation of Irs- 1 in muscle (42), and $\operatorname{Pkc} \theta$ activity was found to be increased in the skeletal muscle of the humanized resistin mice (Figure 4E). No difference was seen in intrahepatic triglyceride levels or Lpl expression in the livers of these animals (Supplemental Figure 7).

Increased WAT inflammation and macrophage infiltration in the humanized resistin mice. We also performed gene-expression analysis in WAT after high-fat-diet feeding. The humanized resistin mice had increased expression of several inflammatory markers in WAT, including Tnf- $\alpha$, Il-6, Il-1 $\beta$, Cxcl5, monocyte chemotactic protein-1 (Mcp-1), and Cd68 (Figure 5A). This is reminiscent of the inflammation seen in humans during obesity, which has been shown to contribute to the insulin resistance that accompanies this condition (43-45). In addition, there was a modest but significant decrease

\section{Figure 5}

Inflamed WAT in humanized resistin mice. (A) Gene-expression analysis in WAT of humanized resistin and control mice. (B) Immunohistochemical detection of the macrophage-specific antigen F4/80 (white arrows) in epididymal adipose tissue from humanized resistin and control mice. Original magnification, $\times 200$ (upper panels); $\times 400$ (lower panels). (C) Ser307 phosphorylation levels of Irs-1 in WAT normalized to controls. ${ }^{*} P<0.05 ;{ }^{* *} P<0.01$. Data are expressed as mean \pm SEM; $n=9$.

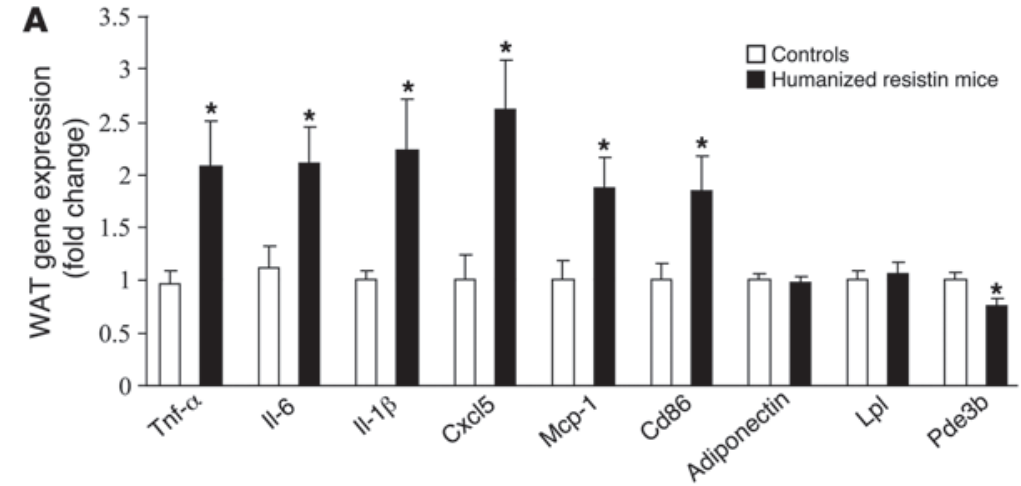

B
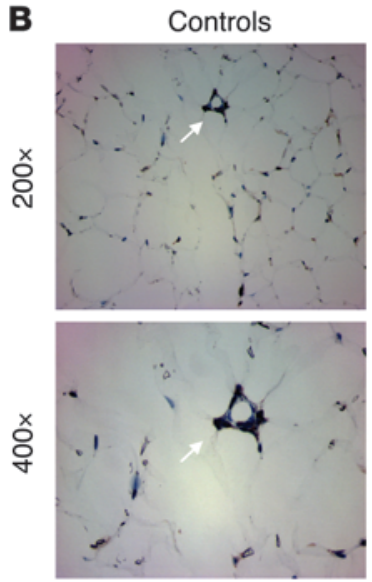

Humanized resistin mice
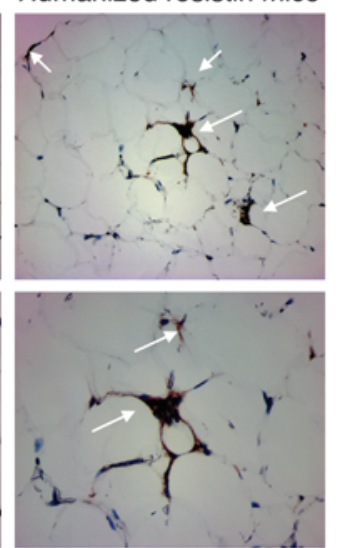

C WAT Irs-1 phosphorylation

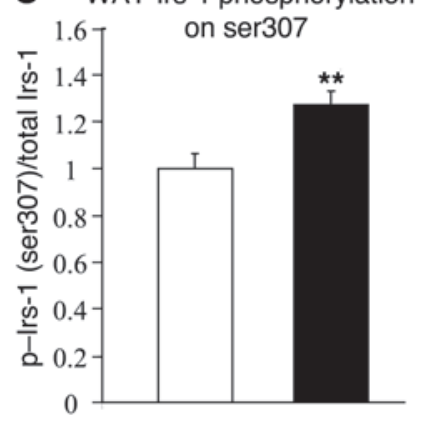




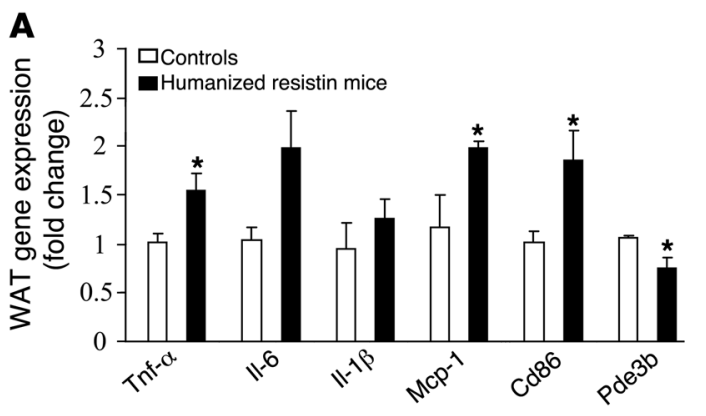

B

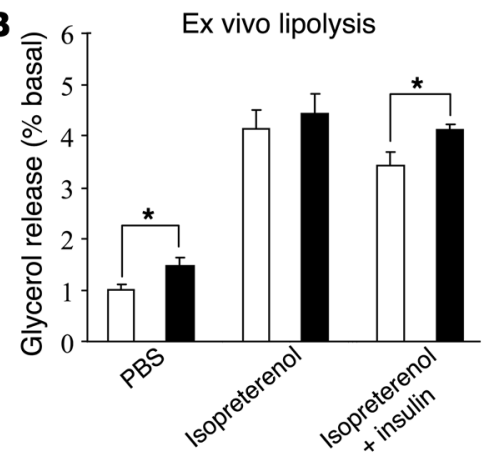

C

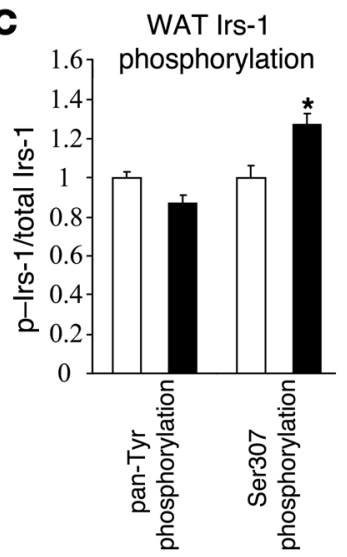

D

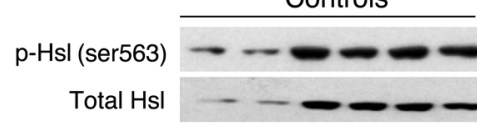

E

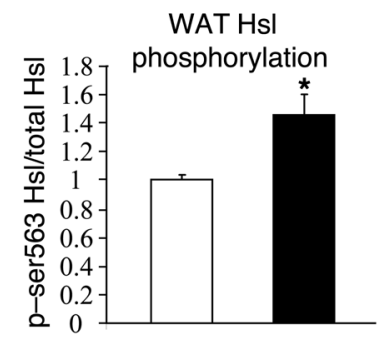

inflammation, ser307 phosphorylation of Irs- 1 was increased in the WAT of humanized resistin mice (Figure 5C).

Adipose tissue inflammation and increased lipolysis precede the skeletal muscle changes in humanized resistin mice. Thus far, we have shown that after 2-3 weeks on the high-fat diet, the humanized resistin mice had increased inflammation in WAT as well as skeletal muscle insulin resistance. To address the question of which of these metabolically deleterious effects of human resistin are primary, humanized resistin mice were evaluated after only 4 days on the high-fat diet. Remarkably, even at this early time, several inflammation markers were already increased in the WAT of humanized resistin mice, including Tnf- $\alpha$, Mcp-1, and the macrophage marker Cd68 (Figure 6A). Also at this time, serum FFAs and glycerol were higher in the humanized resistin mice (Table 2), suggestive of increased lipolysis in these animals. Consistently, ex vivo analysis of WAT from the humanized resistin mice revealed increased basal lipolysis with reduced suppression by insulin (Figure 6B). This insulin resistance was paralleled by reduced tyrosine phosphorylation of Irs- 1 and increased inhibitory serine phosphorylation of Irs-1 in the WAT of the humanized resistin mice (Figure 6C). Among the cytokines increased in the inflamed WAT of humanized resistin mice, Tnf- $\alpha$ in particular is known to induce lipolysis by suppressing expression of Pde $3 b$, thereby activating Hsl by increasing its phosphorylation $(52,53)$. Indeed, Hsl phosphorylation on ser563, an index of HSL activity, was increased in humanized resistin mice (Figure 6, D and $\mathrm{E}$, respectively). In contrast, at these early times, no difference was observed in serum triglyceride levels, muscle Lpl expression, muscle triglyceride levels, or muscle DAG levels (Table 2 and Supplemental Figure 8, respectively). In hyperinsulinemiceuglycemic clamp studies, the humanized resistin mice showed a tendency toward lower GIR and lower rate of disposal, but this difference did not reach statistical significance (Supplemental Figure 9). Thus, increased inflammation and lipolysis in WAT precedes the insulin resistance and the accumulation of lipid in skeletal muscle of the humanized resistin mice.

High-fat diet increases human resistin in WAT. We next assessed the mechanism by which high-fat feeding exacerbates the insulin resistance phenotype in the humanized resistin mice. In control mice, the expression of a macrophage-specific marker, Cd68, was increased during both short-term (4 days) and longer-term (3 weeks) high-fat feeding (Figure 7A). In the humanized resistin mice, high-fat feeding increased WAT Cd68 expression much more dramatically (Figure 7A). As expected, this increase in WAT macrophage infiltration led to a significant increase in the expression of human resistin in the WAT of the humanized resistin mice on short-term (5-fold) and longer-term (11.7-fold) high-fat feeding compared with normal-chow-fed animals (Figure 7B). These data are consistent with the conclusion that high-fat feeding increases WAT macrophage infiltration, and this is markedly increased when the macrophages express human resistin.

\section{Table 2}

Plasma metabolic profile of humanized resistin mice on high-fat diet for 4 days

$\begin{array}{lcc}\text { Genotype } & \text { Control mice } & \text { Humanized resistin mice } \\ \text { TGs }(\mathrm{mg} / \mathrm{dl}) & 52.2 \pm 3.5 & 53.9 \pm 3.9 \\ \text { NEFA }(\mathrm{mEq} / \mathrm{l}) & 0.41 \pm 0.03 & 0.57 \pm 0.02^{\mathrm{A}} \\ \text { Glycerol }(\mathrm{mg} / \mathrm{dl}) & 0.021 \pm 0.002 & 0.031 \pm 0.003^{\mathrm{A}}\end{array}$

Humanized resistin mice show serum profile indicative of increased lipolysis. NEFA, nonesterified fatty acids. Data are expressed as mean $\pm \mathrm{SEM} ; n=8$. ${ }^{\mathrm{A}} P<0.05$. 

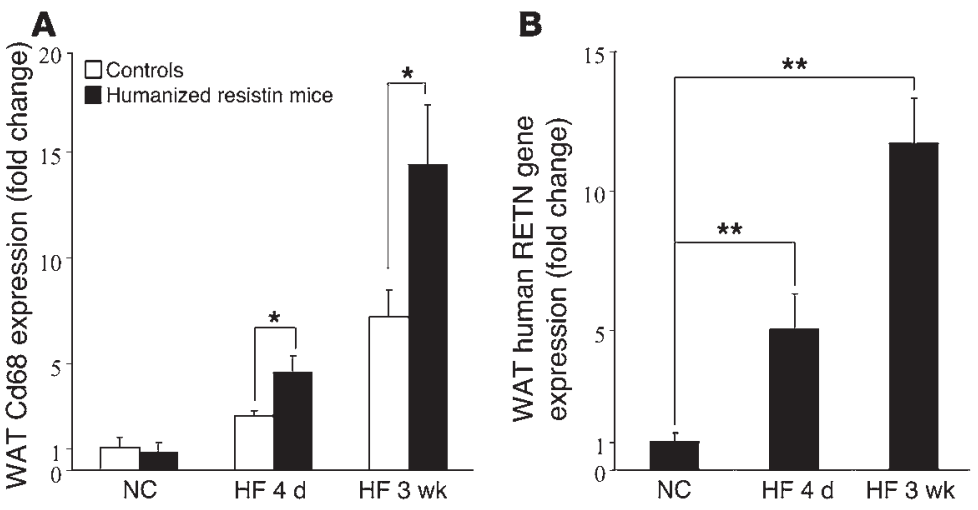

\section{Figure 7}

High-fat feeding increases WAT macrophage infiltration and human resistin expression in humanized resistin mice. (A) Gene-expression analysis of Cd68 in WAT of humanized resistin and control mice on high-fat diet normalized to the expression in mice on normal chow. (B) Expression of human RETN in WAT of humanized resistin mice on high-fat diet normalized to its expression in WAT of mice on normal chow. NC, normal-chow diet; HF, high-fat diet. ${ }^{*} P<0.05$; ${ }^{* \star} P<0.01$. Data are expressed as mean \pm SEM; $n=5-9$.

\section{Discussion}

While there is very strong evidence that hyperresistinemia causes insulin resistance and predisposes to T2DM in rodents (11-15), the role of resistin in humans is still uncertain, especially because in humans circulating resistin is mostly derived from macrophages rather than adipocytes $(24-28,30)$. Although this might suggest a divergent role for resistin in humans, the present study clearly demonstrates that macrophage-derived human resistin promotes insulin resistance in mice fed high-fat diets, much as adipocytederived mouse resistin does in wild-type mice. This study thus extends our understanding of resistin pathophysiology in humans in 2 important ways. First, this is the first study, to our knowledge, that shows that macrophage-derived human resistin contributes to insulin resistance in vivo. Second, this study identifies the proinflammatory property of human resistin as a major contributor to its role in insulin resistance. In this regard, we postulate that human resistin constitutes an important link between innate immunity and glucose metabolism, linking well-documented obesity-induced inflammation to insulin resistance.

We find that macrophage-derived human resistin leads to insulin resistance that mainly affects glucose uptake into muscle. Humanized resistin mice have elevated intramuscular triglycerides, which are associated with decreased insulin sensitivity. This is concomitant with increased intracellular lipid products, notably DAG, which is a strong activator of $\operatorname{Pkc} \theta$, a kinase that catalyzes the inhibitory serine phosphorylation of Irs-1 and hence decreases Irs- 1 tyrosine phosphorylation to promote insulin resistance $(54,55)$. This pathway is clearly activated in the skeletal muscle of humanized resistin mice, which exhibits increased DAG, activation of $\mathrm{Pkc} \theta$, and decreased tyrosine phosphorylation of Irs- 1 . These changes in the humanized resistin mice are likely a consequence of increased muscle expression of Lpl, which is induced by high-fat diet in humans (56) and has been shown to lead to triglyceride accumulation and activation of the $\mathrm{Pkc} \theta$ pathway to insulin resistance (41). Moreover, increased skeletal muscle expression of Lpl has been shown to reduce serum triglyceride levels (57), which plausibly explains the lower serum triglycerides seen in the humanized resistin mice. It should be noted that resistin has been previously shown to increase LPL activity in adipocytes (58).

It has been postulated that circulating FFAs might induce Lpl as well as directly promote intramyocellular DAG production and the activation of the $\operatorname{Pkc} \theta$ signaling pathway $(7,40,59)$. Indeed, we suspect that elevated serum FFAs are a primary cause of insulin resistance in the humanized resistin mice. Shortly after onset of high-fat feeding, we did not observe changes in skeletal muscle Lpl expression or activation of the $\operatorname{Pkc} \theta$ pathway, whereas we did note changes in serum FFAs and glycerol secondary to increased adipose tissue lipolysis in the humanized resistin mice. Several recent reports implicate resistin in the control of lipolysis, although the mechanism is not clear (60-63). In the humanized resistin mice, we found increased activity of Hsl, a major lipase controlling lipolysis in WAT. Moreover, our data suggest that the mechanism by which macrophage-derived resistin increases HSL activity is by inducing WAT inflammation, which occurs shortly after high-fat feeding of the humanized resistin mice. Tnf- $\alpha$, one of the inflammatory mediators induced in the WAT of humanized resistin mice, activates lipolysis by suppressing Pde3b, the major enzyme involved in cAMP degradation and hence attenuation of PKA activity (52). Pde $3 \mathrm{~b}$ expression is decreased in the adipose tissue of the humanized resistin mice, and this correlates with enhanced phosphorylation of Hsl at its activating PKA site.

Our finding that macrophage-derived human resistin exacerbates murine insulin resistance is of interest given the approximately $60 \%$ sequence identity between mouse and human resistin, which is less than is typical of other metabolic hormones, such as leptin and insulin. On the other hand, parathyroid hormone (PTH) is $70 \%$ identical between mouse and human, and PTH-related polypeptide shares less than $30 \%$ identity with PTH, yet both mouse and human PTH and PTH-related polypeptide bind to the same receptor $(64,65)$. Another example is FGF19 and its mouse homo$\log$ Fgf15, which have similar functions despite the modest (53\%) sequence identity (66). This could be attributed to the similarity in the structures of these molecules. Thus, although the receptor for resistin is unknown, there is precedent for molecules with this level of conservation to have similar functions across species.

The similar phenotypes of the 2 lines of humanized resistin mice with 3- to 4-fold differences in the extent of their hyperresistinemia may suggest that the putative resistin receptor is saturated at both concentrations. This finding is reminiscent of the plateau in resistin action observed after infusion in the rat, where others have observed similar metabolic effects when serum resistin levels were elevated 2 -fold versus 15 -fold (13). Thus, the effects of circulating resistin may be saturating at twice the normal level. In addition, the macrophage-derived human resistin may also function locally, and thus serum resistin is likely not the entire contributor to the phenotypic effects of macrophage expression of human resistin.

In sum, we have utilized what we believe is a novel mouse model of human hyperresistinemia to demonstrate that macrophagederived human resistin exacerbates high-fat-diet-induced insulin resistance. The increase in local WAT human resistin expression 
created by high-fat diet is the major driver of this phenotype. Although the insulin resistance is highly manifested in skeletal muscle, the primary effect of macrophage production of human resistin is inflammation of WAT that is reminiscent of the inflammation seen in human obesity. The macrophage infiltration triggered by high-fat feeding is augmented by a resistin-dependent increase in the chemoattractant Mcp-1, leading to increased inflammation as well as lipolysis which, through increased FFAs, leads to muscle lipid accumulation, activation of the $\operatorname{Pkc} \theta$ pathway, and insulin resistance. The effect of macrophage-derived human resistin in promoting and maintaining an inflammatory environment is thus likely to be a factor contributing to metabolic syndrome in humans with increased adiposity. The humanized resistin mice represent a unique model for understanding the pathophysiology associated with increased macrophage-derived human resistin and developing therapies to counter its effects and ameliorate insulin resistance and diabetes.

\section{Methods}

Derivation and treatment of mice. For the derivation of mice that overexpress human resistin in monocyte/macrophage lineage, the coding region of human resistin was obtained by PCR from $\mathrm{pZac} 2.1$ human resistin vector, which contains the human resistin cDNA (11). The human resistin cDNA was then subcloned into the pCD68-hSR-A construct after excision of the hSR-A cDNA (a generous gift from David Greaves, Sir William Dunn School of Pathology) (36). The fusion construct containing human resistin, $2.9 \mathrm{~kb}$ hCD68 promoter, IVS1, and bGH polyadenylation signal was excised using SalI and BsaAI and then injected into the pronucleus of fertilized C57BL/6 mouse eggs (Transgenic and Chimeric Mouse Facility, University of Pennsylvania). Transgenic founder mice (designated CD68hR) were identified by PCR of tail DNA with the forward primer covering the $3^{\prime}$ end of the 2.9-kb hCD68 region (5'-CTGGGTTGCTAACCATCTCC-3') and the reverse primer covering the $5^{\prime}$-end of the human resistin cDNA (5'-CAGGCCAATGCTGCTTATTG-3'). Positive mice were then propagated by mating to Retn ${ }^{-/-}$mice (16). The progeny $\left(\right.$Retn $\left.^{+/-C D 68 h R}\right)$ were then crossed back to the Retn ${ }^{-/}$mice to produce the humanized resistin mice that express human resistin without expressing murine resistin (Retn ${ }^{-/}$ CD68hR). Retn ${ }^{-/-}$littermates were used as controls in this study. Animals used in all experiments were age-matched (9- to 10-week-old) male mice housed in groups of 4 or 5 in plastic microisolator cages at $22^{\circ} \mathrm{C}$ with 12-hour light/12-hour dark cycle and had free access to food and water. Unless otherwise stated, all animals were put on a high-fat diet (60\% fat diet, D12492; Research Diets Inc.) for 2-3 weeks. All protocols for animal use and euthanasia were approved by the Animal Care Committee at the University of Pennsylvania and were in accordance with NIH guidelines.

$R N A$ preparation and quantitative PCR analysis. Mice were killed and their tissues isolated and immediately placed in liquid nitrogen for later use. Total RNA from tissues was isolated using RNeasy kit (QIAGEN) according to the manufacturer's instructions. RNA integrity was determined with UV spectrophotometry. Reverse transcription was performed using the High Capacity cDNA Reverse Transcription Kit (Applied Biosystems) according to the manufacturer's instructions. This cDNA served as the template for real-time (TaqMan) quantitative PCR, using the ABI Prism 7900 sequence detection system (Applied Biosystems). The housekeeping gene 36B4 was used to normalize results to starting amounts of cDNA. All primer/probe sets were purchased as assay-on-demand sets (Applied Biosystems). All reactions were performed using TaqMan Universal PCR Master Mix (Applied Biosystems) and were evaluated using the standard curve method.

Serum metabolic profile analysis. All serum measurements were done on mice after an overnight fast unless otherwise indicated. Glucose levels were deter- mined using a standard glucometer (LifeScan). Serum nonesterified fatty acids were determined using the NEFA Determination Kit (Wako Diagnostics). Serum triglycerides and glycerol were measured with the Serum Triglyceride and Glycerol Determination Kit (Sigma-Aldrich). Serum adipokines were determined using Mouse Serum Adipokine LINCOplex Kit (Millipore). Nonfasted insulin levels were analyzed using an insulin ELISA kit (Crystal Chem Inc.).

GTT and insulin-tolerance test. GTT was performed after an overnight (16-hour) fast. Blood glucose concentrations were measured before and 15, 30, 45, 60, 90, and 120 minutes after an i.p. injection of dextrose dissolved in PBS $(1 \mathrm{~g} / \mathrm{kg})$. Ultra Sensitive Mouse Insulin ELISA Kit (Crystal Chem Inc.) was used to measure insulin levels during the GTT. Insulin-tolerance testing was carried out in animals that were fasted for 6 hours, beginning at approximately $9 \mathrm{am}$. After an i.p. bolus injection of recombinant human regular insulin $(0.75 \mathrm{U} / \mathrm{kg}$; Lilly), blood glucose concentrations were measured at 15 -minute intervals as described above.

Hyperinsulinemic-englycemic clamp. Hyperinsulinemic-euglycemic clamp analysis was performed as described previously (11), with minor modifications. In brief, 4-5 days before the clamp, mice were anesthetized with sodium pentobarbital ( $80-100 \mathrm{mg} / \mathrm{kg}$ i.p.), and a catheter was inserted into the right internal jugular vein for infusion. After recovery, the mice were fasted for 4 hours, placed in restrainers, and administered a bolus injection of $5 \mu \mathrm{Ci}$ of $[3-3 \mathrm{H}]$ glucose followed by continuous i.v. infusion at $0.05 \mu \mathrm{Ci}$ / min. Baseline glucose kinetics was measured for 60 minutes followed by hyperinsulinemic clamp for 120 minutes. A priming dose of regular insulin (16 mU/kg; Lilly) was given i.v. followed by continuous infusion at $2.5 \mathrm{mU} /$ $\mathrm{kg} / \mathrm{min}$, which resulted in insulin levels of $8.9 \pm 1.6$ and $9.3 \pm 3.0 \mathrm{ng} / \mathrm{ml}$ for the controls and the humanized resistin mice, respectively. Blood glucose was maintained at $120-140 \mathrm{mg} / \mathrm{dl}$ via a variable GIR of $20 \%$. The actual blood glucose levels were $135.8 \pm 4.3$ and $134.3 \pm 2.9 \mathrm{mg} / \mathrm{dl}$ for the controls and the humanized resistin mice, respectively. Ten $\mu \mathrm{Ci} 2$-deoxy-D[1-14C] glucose was injected 45 minutes before the end of the clamp, and blood samples were collected to estimate glucose uptake. The mice were euthanized, and liver, perigonadal WAT, and soleus muscle were excised, frozen immediately in liquid nitrogen, and stored at $-80^{\circ} \mathrm{C}$ for subsequent analysis of glucose uptake and other analysis.

Phosphorylation of Irs-1. Muscle and WAT tissue were homogenized with T-PER Tissue Protein Extraction Reagent in the presence of protease and phosphatase inhibitors (Pierce Protein Research Products; Thermo Scientific). Total IRS-1, phospho-IRS-1 (ser307), and phospho-IRS-1 pan tyrosine (pan-Tyr) were determined using PathScan Sandwich ELISA Kit according to manufacturer's instructions (Cell Signaling Technology).

Immunohistochemistry. Adipose tissue samples were fixed for 12-16 hours at room temperature in formalin fixative (Sigma-Aldrich) and embedded in paraffin. Five-micrometer sections cut at 50-mm intervals were mounted on charged glass slides, deparaffinized in xylene, and stained for expression of F4/80 as described elsewhere (43). A rat anti-mouse monoclonal antibody was used to detect the F4/80 antigen (Invitrogen).

Akt and HSL phosphorylation and measurement of $P k c \theta$ activity. $\mathrm{Pkc} \theta$ membrane translocation assays were performed according to methods previously described (67). In brief, $50 \mu \mathrm{g}$ of crude membrane and cytosol muscle protein extracts were resolved by SDS-PAGE using an $8 \%$ gel and electrotransferred onto PVDF (Millipore). The membrane was then blocked for 2 hours at room temperature in PBS-Tween-20 (10 $\mathrm{mM} \mathrm{NaH}_{2} \mathrm{PO}_{4}, 80 \mathrm{mM}$ $\mathrm{Na}_{2} \mathrm{HPO}_{4}, 0.145 \mathrm{mM} \mathrm{NaCl}$, and $0.1 \%$ Tween 20, pH 7.4) containing 5\% (w/v) nonfat dried milk, washed twice, and then incubated overnight with rabbit anti-peptide antibody against $\mathrm{Pkc} \theta$ (Santa Cruz Biotechnology Inc.) diluted 1:100 in rinsing solution. After further washings, membranes were incubated with horseradish peroxidase-conjugated IgG fraction of goat anti-rabbit IgG (Amersham) diluted 1:5,000 in PBS-Tween-20 for 2 hours. Pkc $\theta$ was visual- 
ized on KODAK BioMax MR Film using the ECL+ Chemiluminescence Kit (GE Healthcare). The film was then scanned (Canon), and bands were quantified using Adobe Photoshop Creative Suite software, version 2.0. Membrane translocation of $\mathrm{Pkc} \theta$ was expressed as the ratio of membrane bands to cytosol bands (arbitrary units). For Hsl phosphorylation, WAT protein extracts $(5 \mu \mathrm{g})$ were resolved on SDS-PAGE using a $14 \%$ gel and electrotransferred onto PVDF as mentioned above. Phospho-Hsl antibody was diluted 1:1000 and incubated overnight (Cell Signaling Technology). Phospho-Hsl and total Hsl were visualized and quantified as mentioned above. Western blotting was also used to measure muscle Akt phosphorylation using phospho-specific and total Akt antibodies (Cell Signaling Technology).

Tissue triglycerides and DAG levels. To extract lipid from muscle, $100 \mathrm{mg}$ of muscle tissue was homogenized in chloroform/methanol (2:1), and the resulting homogenate was centrifuged. The lower phase containing the lipid was dried down completely under a stream of $\mathrm{N}_{2}$ and resuspended uniformly in PBS/Triton X-100 solution. Muscle triglycerides levels were determined as described for the serum samples. For DAG levels, samples of $100 \mathrm{mg}$ of muscle tissue were submitted to the Mouse Metabolic Phenotyping Center at Vanderbilt University Medical Center (Nashville, Tennessee, USA).

Ex vivo lipolysis. Epididymal fat pads were isolated from mice and weighed. Each pad was cut into 3 equal pieces and incubated in Krebs-Ringer Bicarbonate Buffer containing 1\% fatty acid-free BSA (Sigma-Aldrich). The samples were treated with either PBS or isoproterenol $(1 \mu \mathrm{M})$ or pretreated with insulin $(10 \mu \mathrm{M})$ for 10 minutes before isoproterenol treatment. Samples were incubated at $37^{\circ} \mathrm{C}$ with mild shaking at $150 \mathrm{~g}$. After $120 \mathrm{~min}$ utes, glycerol release was measured using Glycerol Determination Kit; fat pads were homogenized in PBS, and total triglycerides in each sample were assayed using the Triglyceride Determination Kit (Sigma-Aldrich). Glycerol levels were then normalized to total triglyceride levels in each sample.

Muscle Lpl activity. Muscle tissue (50 mg) was isolated and incubated in ice-cold DMEM with $2 \%$ BSA and 2 units/ml heparin (Sigma-Aldrich). The tissue was then homogenized and incubated at $37^{\circ} \mathrm{C}$ for 1 hour. Samples were then centrifuged at $13,000 \mathrm{~g}$ for 10 minutes, and Lpl activity in the supernatant was analyzed using QuantiChrom Lipase Assay Kit (BioAssay Systems). Lipase activity was normalized to total protein in each sample.

Isolation of the SVF and adipocyte fraction of adipose tissue. Mouse epididymal fat pads were minced and digested for 60 minutes at $37^{\circ} \mathrm{C}$ with collagenase II
( $1 \mathrm{mg} / \mathrm{ml})$ (Sigma-Aldrich) in PBS. After filtration of the digested tissue through nylon mesh $(100 \mu \mathrm{m})$, the filtrate was centrifuged at $200 \mathrm{~g}$ for 5 minutes. The SVF and adipocyte fraction were obtained from the resulting pellet and supernatant, respectively. RNA isolation and quantitative PCR were performed as described earlier.

Nuclear MRI. Nuclear MRI analysis was done at the Mouse Phenotyping, Physiology and Metabolism Core, University of Pennsylvania School of Medicine, as described elsewhere (11).

Statistics. Values are reported as mean \pm SEM. Pairwise comparisons of quantitative phenotypes between mice of different genotypes were assessed by 2 -tailed Student's $t$ test assuming unequal variance. Values of $P<0.05$ were accepted as statistically significant.

\section{Acknowledgments}

We thank C. Steppan and J. Wang for help with the initial Socs3 studies and $\mathrm{K}$. Kaestner for critically reading the manuscript. We are grateful to the Penn Diabetes and Endocrinology Research Center (NIH P30 DK19525) for providing outstanding core services, and we particularly thank J. Richa of the Transgenic and Chimeric Mouse Core, H. Collins of the Radioimmunoassay/Biomarkers Core, and R. Dhir of the Mouse Phenotyping, Physiology and Metabolism Core (also supported by NIDDK P01 DK49210) for their technical help. We also thank the Mouse Metabolic Phenotyping Center at Vanderbilt University Medical Center (NIH P30 DK59637) for the DAG assays. This work was supported by the National Institute of Diabetes and Digestive and Kidney Diseases (NIDDK P01 DK49210 to M.A. Lazar). M. Qatanani was supported by an ADA-Takeda Mentor-Based Research Fellowship. D.R. Greaves is supported by the British Heart Foundation.

Received for publication August 26, 2008, and accepted in revised form December 8, 2008.

Address correspondence to: Mitchell A. Lazar, University of Pennsylvania School of Medicine, 700 CRB, 415 Curie Blvd., Philadelphia, Pennsylvania 19104-6149, USA. Phone: (215) 898-0198; Fax: (215) 898-5408; E-mail: lazar@mail.med.upenn.edu.
1. Li, Z., Bowerman, S., and Heber, D. 2005. Health ramifications of the obesity epidemic. Surg. Clin. North Am. 85:681-701.

2. Olshansky, S.J. 2005. Projecting the future of U.S. health and longevity. Health Aff. (Millwood). 24(Suppl. 2):W5R86-W5R89.

3. American Diabetes Association. 2007. Total prevalence of diabetes and pre-diabetes. http://www. diabetes.org/diabetes-statistics/prevalence.jsp.

4. Zimmet, P., Alberti, K.G., and Shaw, J. 2001. Global and societal implications of the diabetes epidemic. Nature. 414:782-787.

5. Kahn, B.B., and Flier, J.S. 2000. Obesity and insulin resistance. J. Clin. Invest. 106:473-481.

6. Flier,J.S. 2004. Obesity wars: molecular progress confronts an expanding epidemic. Cell. 116:337-350.

7. Qatanani, M., and Lazar, M.A. 2007. Mechanisms of obesity-associated insulin resistance: many choices on the menu. Genes Dev. 21:1443-1455.

8. Lazar, M.A. 2007. Resistin- and obesity-associated metabolic diseases. Horm. Metab. Res. 39:710-716.

9. Holcomb, I.N., et al. 2000. FIZZ1, a novel cysteinerich secreted protein associated with pulmonary inflammation, defines a new gene family. $E M B O J$. 19:4046-4055.

10. Kim, K.H., Lee, K., Moon, Y.S., and Sul, H.S. 2001. A cysteine-rich adipose tissue-specific secretory factor inhibits adipocyte differentiation. J. Biol. Chem. 276:11252-11256.
11. Steppan, C.M., et al. 2001. The hormone resistin links obesity to diabetes. Nature. 409:307-312.

12. Rajala, M.W., et al. 2004. Regulation of resistin expression and circulating levels in obesity, diabetes, and fasting. Diabetes. 53:1671-1679.

13. Rajala, M.W., Obici, S., Scherer, P.E., and Rossetti, L. 2003. Adipose-derived resistin and gut-derived resistin-like molecule-beta selectively impair insulin action on glucose production. J. Clin. Invest. 111:225-230.

14. Satoh, H., et al. 2004. Adenovirus-mediated chronic "hyper-resistinemia" leads to in vivo insulin resistance in normal rats. J. Clin. Invest. 114:224-231.

15. Rangwala, S.M., et al. 2004. Abnormal glucose homeostasis due to chronic hyperresistinemia. Diabetes. 53:1937-1941

16. Banerjee, R.R., et al. 2004. Regulation of fasted blood glucose by resistin. Science. 303:1195-1198.

17. Muse, E.D., et al. 2004. Role of resistin in dietinduced hepatic insulin resistance. J. Clin. Invest. 114:232-239.

18. Kim, K.H., Zhao, L., Moon, Y., Kang, C., and Sul, H.S. 2004. Dominant inhibitory adipocyte-specific secretory factor (ADSF)/resistin enhances adipogenesis and improves insulin sensitivity. Proc. Natl. Acad. Sci. U. S. A. 101:6780-6785.

19. Steppan, C.M., Wang, J., Whiteman, E.L., Birnbaum, M.J., and Lazar, M.A. 2005. Activation of SOCS-3 by resistin. Mol. Cell. Biol. 25:1569-1575.
20. Qi, Y., et al. 2006. Loss of resistin improves glucose homeostasis in leptin deficiency. Diabetes. 55:3083-3090.

21. McTernan, C.L., et al. 2002. Resistin, central obesity, and type 2 diabetes. Lancet. 359:46-47.

22. McTernan, P.G., et al. 2002. Increased resistin gene and protein expression in human abdominal adipose tissue. J. Clin. Endocrinol. Metab. 87:2407.

23. Vidal-Puig, A., and O'Rahilly, S. 2001. Resistin: a new link between obesity and insulin resistance? Clin. Endocrinol. (Oxf.) 55:437-438.

24. Wang, H., Chu, W.S., Hemphill, C., and Elbein, S.C. 2002. Human resistin gene: molecular scanning and evaluation of association with insulin sensitivity and type 2 diabetes in Caucasians. J. Clin. Endocrinol. Metab. 87:2520-2524.

25. Smith, S.R., Bai, F., Charbonneau, C., Janderova, L., and Argyropoulos, G. 2003. A promoter genotype and oxidative stress potentially link resistin to human insulin resistance. Diabetes. 52:1611-1618.

26. Osawa, H., et al. 2005. Resistin SNP-420 determines its monocyte mRNA and serum levels inducing type 2 diabetes. Biochem. Biophys. Res. Commun. 335:596-602

27. Osawa, H., et al. 2004. The G/G genotype of a resistin single-nucleotide polymorphism at -420 increases type 2 diabetes mellitus susceptibility by inducing promoter activity through specific binding of Sp1/3. Am. J. Hum. Genet. 75:678-686. 
28. Janke, J., Engeli, S., Gorzelniak, K., Luft, F.C., and Sharma, A.M. 2002. Resistin gene expression in human adipocytes is not related to insulin resistance. Obes. Res. 10:1-5.

29. Kielstein, J.T., Becker, B., Graf, S., Brabant, G., Haller, H., and Fliser, D. 2003. Increased resistin blood levels are not associated with insulin resistance in patients with renal disease. Am. J. Kidney Dis. 42:62-66.

30. Patel, L., et al. 2003. Resistin is expressed in human macrophages and directly regulated by PPAR gamma activators. Biochem. Biophys. Res. Commun. 300:472-476.

31. Lehrke, M., et al. 2004. An inflammatory cascade leading to hyperresistinemia in humans. PLoS Med. 1:e45.

32. Pang, S.S., and Le, Y.Y. 2006. Role of resistin in inflammation and inflammation-related diseases. Cell. Mol. Immunol. 3:29-34.

33. Reilly, M.P., et al. 2005. Resistin is an inflammatory marker of atherosclerosis in humans. Circulation. 111:932-939.

34. Senolt, L., et al. 2007. Resistin in rheumatoid arthritis synovial tissue, synovial fluid and serum. Ann. Rheum. Dis. 66:458-463.

35. Savage, D.B., et al. 2001. Resistin / Fizz3 expression in relation to obesity and peroxisome proliferatoractivated receptor-gamma action in humans. Diabetes. 50:2199-2202.

36. Gough, P.J., Gordon, S., and Greaves, D.R. 2001. The use of human CD68 transcriptional regulatory sequences to direct high-level expression of class A scavenger receptor in macrophages in vitro and in vivo. Immunology. 103:351-361.

37. Axelsson, J., et al. 2006. Elevated resistin levels in chronic kidney disease are associated with decreased glomerular filtration rate and inflammation, but not with insulin resistance. Kidney Int. 69:596-604.

38. Piestrzeniewicz, K., et al. 2008. Resistin increases with obesity and atherosclerotic risk factors in patients with myocardial infarction. Metabolism. 57:488-493.

39. Fain, J.N. 2006. Release of interleukins and other inflammatory cytokines by human adipose tissue is enhanced in obesity and primarily due to the nonfat cells. Vitam. Horm. 74:443-477.

40. Zechner, R., et al. 2000. The role of lipoprotein lipase in adipose tissue development and metabolism. Int. J. Obes. Relat. Metab. Disord. 24(Suppl. 4):S53-S56.

41. Kim, J.K., et al. 2001. Tissue-specific overexpression of lipoprotein lipase causes tissue-specific insulin resistance. Proc. Natl. Acad. Sci.U. S. A. 98:7522-7527.
42. Petersen, K.F., and Shulman, G.I. 2006. Etiology of insulin resistance. Am. J. Med. 119:S10-S16.

43. Weisberg, S.P., et al. 2006. CCR2 modulates inflammatory and metabolic effects of high-fat feeding. J. Clin. Invest. 116:115-124.

44. Weisberg, S.P., et al. 2003. Obesity is associated with macrophage accumulation in adipose tissue. J. Clin. Invest. 112:1796-1808.

45. Xu, H., et al. 2003. Chronic inflammation in fat plays a crucial role in the development of obesity-related insulin resistance. J. Clin. Invest. 112:1821-1830.

46. Rahn Landstrom, T., Mei, J., Karlsson, M., Manganiello, V., and Degerman, E. 2000. Down-regulation of cyclic-nucleotide phosphodiesterase 3B in 3T3$\mathrm{L} 1$ adipocytes induced by tumour necrosis factor alpha and cAMP. Biochem. J. 346:337-343.

47. Kanda, H., et al. 2006. MCP-1 contributes to macrophage infiltration into adipose tissue, insulin resistance, and hepatic steatosis in obesity. J. Clin. Invest. 116:1494-1505.

48. Di Gregorio, G.B., et al. 2005. Expression of CD68 and macrophage chemoattractant protein-1 genes in human adipose and muscle tissues: association with cytokine expression, insulin resistance, and reduction by pioglitazone. Diabetes. 54:2305-2313.

49. Verma, S., et al. 2003. Resistin promotes endothelial cell activation: further evidence of adipokineendothelial interaction. Circulation. 108:736-740.

50. Rui, L., et al. 2001. Insulin/IGF-1 and TNF-alpha stimulate phosphorylation of IRS-1 at inhibitory Ser307 via distinct pathways. J. Clin. Invest. 107:181-189.

51. Gao, Z., et al. 2002. Serine phosphorylation of insulin receptor substrate 1 by inhibitor kappa B kinase complex. J. Biol. Chem. 277:48115-48121.

52. Zhang, H.H., Halbleib, M., Ahmad, F., Manganiello, V.C., and Greenberg, A.S. 2002. Tumor necrosis factor-alpha stimulates lipolysis in differentiated human adipocytes through activation of extracellular signal-related kinase and elevation of intracellular cAMP. Diabetes. 51:2929-2935.

53. Anthonsen, M.W., Ronnstrand, L., Wernstedt, C., Degerman, E., and Holm, C. 1998. Identification of novel phosphorylation sites in hormone-sensitive lipase that are phosphorylated in response to isoproterenol and govern activation properties in vitro. J. Biol. Chem. 273:215-221.

54. Farese, R.V., et al. 2007. Muscle-specific knockout of PKC-lambda impairs glucose transport and induces metabolic and diabetic syndromes. J. Clin. Invest. 117:2289-2301.

55. Zhang, D., et al. 2007. Mitochondrial dysfunc- tion due to long-chain Acyl-CoA dehydrogenase deficiency causes hepatic steatosis and hepatic insulin resistance. Proc. Natl. Acad. Sci. U. S. A. 104:17075-17080.

56. Schrauwen-Hinderling, V.B., et al. 2005. Intramyocellular lipid content and molecular adaptations in response to a 1-week high-fat diet. Obes. Res. 13:2088-2094.

57. Jensen, D.R., et al. 1997. Prevention of dietinduced obesity in transgenic mice overexpressing skeletal muscle lipoprotein lipase. Am. J. Physiol. 273:R683-R689.

58. Kim, S.J., Nian, C., and McIntosh, C.H. 2007. Resistin is a key mediator of glucose-dependent insulinotropic polypeptide (GIP) stimulation of lipoprotein lipase (LPL) activity in adipocytes. J. Biol. Chem. 282:34139-34147.

59. de Luca, C., and Olefsky, J.M. 2008. Inflammation and insulin resistance. FEBS Lett. 582:97-105.

60. Ort, T., et al. 2005. Recombinant human FIZZ3/ resistin stimulates lipolysis in cultured human adipocytes, mouse adipose explants, and normal mice. Endocrinology. 146:2200-2209.

61. Pravenec, M., et al. 2006. Fat-specific transgenic expression of resistin in the spontaneously hypertensive rat impairs fatty acid re-esterification. Int.J. Obes. (Lond.) 30:1157-1159.

62. Pravenec, M., et al. 2003. Transgenic and recombinant resistin impair skeletal muscle glucose metabolism in the spontaneously hypertensive rat. J. Biol. Chem. 278:45209-45215.

63. Rae, C., Robertson, S.A., Taylor, J.M., and Graham, A. 2007. Resistin induces lipolysis and re-esterification of triacylglycerol stores, and increases cholesteryl ester deposition, in human macrophages. FEBS Lett. 581:4877-4883.

64. Guerreiro, P.M., Renfro, J.L., Power, D.M., and Canario, A.V. 2007. The parathyroid hormone family of peptides: structure, tissue distribution, regulation, and potential functional roles in calcium and phosphate balance in fish. Am. J. Physiol. Regul. Integr. Comp. Physiol. 292:R679-R696.

65. He, B., et al. 2002. The murine gene encoding parathyroid hormone: genomic organization, nucleotide sequence and transcriptional regulation. J. Mol. Endocrinol. 29:193-203.

66. Jones, S. 2008. Mini-review: endocrine actions of fibroblast growth factor 19. Mol. Pharm. 5:42-48.

67. Neschen, S., et al. 2005. Prevention of hepatic steatosis and hepatic insulin resistance in mitochondrial acyl-CoA:glycerol-sn-3-phosphate acyltransferase 1 knockout mice. Cell Metab. 2:55-65. 